\title{
Riesgo de enfermedades transmitidas por mosquitos Aedes en la zona metropolitana del Valle de México y amenaza de sindemias en el país
}

\author{
Risk of diseases transmitted by Aedes mosquitoes in the metropolitan area of the Valley \\ of Mexico and the threat of syndemics in the country
}

\author{
José A. Díaz-Quiñonez* \\ Universidad Nacional Autónoma de México, Facultad de Medicina, División de Estudios de Posgrado, Ciudad de México, México
}

...y porque mientras no se lleve a cabo la eliminación de raíz del Aëdes aegypti en América, podrá haber brotes epidémicos urbanos excesivamente costosos que deben ser evitados por la erradicación del vector doméstico en América...

Miguel E. Bustamante

En la nota preliminar del libro Breve historia y antología sobre la fiebre amarilla, de 1964, el poeta, ensayista y dramaturgo Salvador Novo escribió lo siguiente: ${ }^{2}$

\footnotetext{
El día 6 de agosto de 1963, el Dr. don José Álvarez Amézquita, Secretario de Salubridad y Asistencia de México, hizo al Dr. Luther Terry, Surgeon General, Public Health Service, Department of Health U.S.A., un singular obsequio: dentro de un cubito de plástico, le entregó la última pareja -hembra y macho- de Aedes aegypti capturada en nuestro territorio.
}

Aquel evento simbólico representó la apoteosis de una lucha de muchos años para eliminar de nuestro país (y de la Mesoamérica que describe Miguel $\mathrm{E}$. Bustamante), al vector de la fiebre amarilla urbana. Terminaba así una prolongada campaña dirigida por autoridades sanitarias federales y estatales, apoyada por científicos y profesionales de la salud pública. Un programa de salud que facilitó el legítimo derecho de la participación social mediante un enorme trabajo comunitario. En un intercambio epistolar, el secretario
Álvarez Amézquita agradeció al también historiador y cronista, miembro del grupo los Contemporáneos: ${ }^{2}$

Es relevante también y digna de mi reconocimiento, la difusión que a través de la "Historia y Antología de la Fiebre Amarilla" se hará en México de ese problema de salud pública que durante más de 400 años fue una sombra en la conciencia de los hombres responsables en la lucha por un México limpio de los más grandes males.

En septiembre de 1963, durante la sesión plenaria de la XIV Reunión del Consejo Directivo de la Organización Panamericana de la Salud y en el XV del Comité Regional de la Organización Mundial de la Salud se aprobó la Resolución CD14.R13, que concluyó "aceptar el informe presentado por México, felicitar a dicho país por los trabajos realizados, y declarar su territorio libre de Aedes aegypti". En su quinto informe de gobierno, el presidente Adolfo López Mateos comunicó a la nación la erradicación de la enfermedad que, a través del mosquito como vector, durante siglos mantuvo en riesgo sanitario constante a los puertos y ciudades costeras del país. ${ }^{3}$

Empero, esto ya forma parte de nuestra historia, de la historia de la literatura y la salud pública mexicanas.

En 1979, en México se registró la primera epidemia de fiebre por dengue después de la reintroducción de
Gac Med Mex. 2020;156:369-371

Disponible en PubMed

www.gacetamedicademexico.com

0016-3813/๑ 2020 Academia Nacional de Medicina de México, A.C. Publicado por Permanyer. Este es un artículo open access bajo la licencia CC BY-NC-ND (http://creativecommons.org/licenses/by-nc-nd/4.0/). 
Aedes aegypti, el cual es considerado el vector primario asociado a la transmisión de los cuatro serotipos del virus, en tanto que Aedes albopictus, menos eficiente, es considerado el vector secundario. Además de la fiebre amarilla, al género Aedes pertenecen mosquitos transmisores de otras enfermedades como fiebre del Zika, fiebre del Nilo Occidental, diversas encefalitis, artritis epidémica por chikunguña y fiebre de Mayaro.

Aedes es originario de África, pero en la actualidad se encuentra en las regiones tropicales y subtropicales del planeta. Su expansión a diferentes territorios se ha asociado a las migraciones humanas en masa. ${ }^{4}$ Inicialmente, el mosquito llegó a América con el comercio de esclavos africanos entre los siglos XV y XIX, después se diseminó por Asia como resultado del comercio durante los siglos XVIII y XIX; la redistribución mundial sucedió posterior a la Segunda Guerra Mundial, con el movimiento de las tropas bélicas.

Durante los últimos 50 años, las enfermedades transmitidas por Aedes han emergido o reemergido en todo el mundo. ${ }^{5}$ El virus del dengue continúa en aumento y ocasiona aproximadamente 390 millones de infecciones humanas por año, el virus de chikunguña se ha propagado por todo el mundo desde principios de la década de 2000 , el virus del Zika se ha diseminado también durante los últimos cinco años y la fiebre amarilla ha resurgido recientemente en África y Sudamérica. ${ }^{6}$ La expansión de estas enfermedades se explica por la presencia de condiciones que favorecen la dispersión y proliferación de Aedes como el comercio global, el crecimiento de la población, la urbanización no planificada, la implementación ineficiente de programas de control de vectores, de suministro de agua y gestión de desechos, así como la falta de voluntad política y de compromiso comunitario. ${ }^{7}$ Todos los virus que causan estas enfermedades se transmiten principalmente por el mosquito Aedes aegypti y, en menor medida, por Aedes albopictus. Aún no se conoce el impacto económico global de las enfermedades transmitidas por estos vectores, ${ }^{8}$ pero se estima que las pérdidas económicas debidas al dengue son de, al menos, USD 9 mil millones anuales. ${ }^{9}$

En este número 5 de 2020 de Gaceta Médica de México, en dos artículos se revisa la emergencia de enfermedades transmitidas por Aedes. En uno se considera el riesgo que representa el establecimiento del mosquito Aedes aegypti en la zona metropolitana del Valle de México y las enfermedades que puede transmitir en una población de aproximadamente 22 millones de personas. En el otro se dimensiona la sindemia entre COVID-19 y dengue en el sureste de México, región del país que no es la única en riesgo: la primera defunción se registró en El Salto, Jalisco.

En años recientes, la vigilancia entomológica realizada en el Programa de Enfermedades Transmitidas por Vectores ha confirmado la dispersión de Aedes aegypti y su presencia en demarcaciones territoriales de la Ciudad de México ${ }^{10}$ y municipios conurbados, con lo cual se ha demostrado que la presencia del vector no se circunscribe a una altitud y temperatura. ${ }^{11}$

En su revisión, Mejía Guevara et al. reportan la identificación constante y en aumento del vector a lo largo de los últimos cinco años en la zona metropolitana del Valle de México, lo que evidencia el establecimiento del mosquito en las demarcaciones territoriales Azcapotzalco, Coyoacán, Gustavo A. Madero, Iztacalco, Venustiano Carranza y Xochimilco (norte, noreste, sur y centro de la Ciudad de México), ${ }^{12}$ así como en los municipios conurbados Los Reyes La Paz y Nezahualcóyotl, del Estado de México. La oportuna vigilancia entomovirológica realizada por el Instituto de Diagnóstico y Referencia Epidemiológicos "Manuel Martínez Báez" ha confirmado la preocupante presencia de mosquitos infectados por virus del dengue y Zika en algunos especímenes colectados en la Ciudad de México. ${ }^{13}$ La reproducción de mosquitos Aedes aegypti y albopictus es difícil de controlar una vez que se han establecido las poblaciones. Solo se requiere el incremento de la densidad vectorial, una colonización geográfica suficiente y un número adecuado de pacientes infectados para que se presenten brotes (situación que ya sucede en municipios de las zonas bajas del Estado de México y Morelos).

Ante la posibilidad de epidemias por enfermedades transmitidas por este vector, es urgente la comunicación del riesgo a la población en general y la capacitación de los profesionales de la salud para la identificación de casos sospechosos y probables de las arbovirosis transmitidas por Aedes en la zona metropolitana del Valle de México. Sería deseable que el Sistema Nacional de Vigilancia Epidemiológica estableciera la detección rutinaria de otras especies de vectores y otros arbovirus de importancia en salud pública (virus de encefalitis de San Luis, encefalitis equina venezolana, encefalitis del Este, encefalitis del Oeste, fiebre amarilla, virus Mayaro, virus del dengue, del Nilo Occidental, del Zika). Antes de que la dispersión de los vectores sea más extensa, se requiere una intensa campaña de control para proteger a los 
individuos inmunológicamente susceptibles a infecciones primarias por arbovirus.

El término sindemia, introducido recientemente por antropólogos médicos, se refiere a la interacción de dos o más epidemias coexistentes y al exceso de carga de enfermedad resultante. ${ }^{14}$ El término se aplica a problemas de salud sinérgicos que afectan a una población en sus contextos sociales y económicos, los cuales requieren la elaboración de marcos conceptuales específicos para identificar los factores de riesgo, con la finalidad de implementar programas de prevención y control para atender las comorbilidades. ${ }^{15}$

La sindemia entre COVID-19 y dengue en el sureste del país es abordada por Sánchez de la Cruz et al. ${ }^{16}$ Los autores identifican esta sindemia como una amenaza que puede colapsar los sistemas de salud en los estados del sureste y la costa del Pacífico. Estas epidemias simultáneas complican el panorama epidemiológico, así como el diagnóstico, control y tratamiento oportunos. ${ }^{17}$ En México, hasta la semana epidemiológica 38 de 2020, se notificaron 11228 casos de dengue con 28 defunciones. ${ }^{18}$ Ya se han reportado casos de coinfección por los virus dengue y SARS-CoV-2 en distintas entidades federativas, uno de ellos con desenlace fatal en Jalisco. ${ }^{19}$ Este escenario de coinfecciones no solo aplica para dengue, influenza y otras enfermedades infecciosas con un componente estacional, sino también para las epidemias de diabetes y obesidad. La mala noticia es que esta realidad epidemiológica se mantendrá mientras persista la pandemia por COVID-19.

A menos que se adopte un enfoque coordinado y preventivo para controlar al mosquito, los brotes de enfermedades transmitidas por vector serán más comunes y generarán nuevos síndromes en el país (al coincidir con la epidemia de síndromes metabólicos y la pandemia ocasionada por SARS-CoV-2). El mejoramiento de nuestros entornos - a través de una cultura de fomento sanitario y promoción de la saludcontribuirá a una solución a largo plazo para reducir los riesgos sanitarios y la emergencia de enfermedades epidémicas.

\section{Bibliografía}

1. Bustamante ME. La fiebre amarilla en México y su origen en América. México: Secretaría de Salubridad y Asistencia/Instituto de Salubridad y Enfermedades Tropicales; 1958.

2. Novo S. Breve historia y antología sobre la fiebre amarilla. México: Secretaría de Salubridad y Asistencia/Prensa Médica Mexicana; 1964.

3. Informes Presidenciales. Adolfo López Mateos. V Informe de Gobierno. México: Servicio de Investigación y Análisis/Subdirección de Referencia Especializada; 2006.

4. Sherpa S, Rioux D, Goindin D, Fouque F, François O, Després L. At the origin of a worldwide invasion: unraveling the genetic makeup of the Caribbean bridgehead populations of the dengue vector Aedes aegypti. Genome Biol Evol. 2018;10:56-71.

5. Roiz D, Wilson AL, Scott TW, Fonseca DM, Jourdain F, Müller P, et al. Integrated Aedes management for the control of Aedes-borne diseases. PLoS Negl Trop DIS. 2018;12:e0006845.

6. Wilder-Smith A, Gubler DJ, Weaver SC, Monath TP, Heymann DL, Scott TW. Epidemic arboviral diseases: priorities for research and public health. Lancet Infect Dis. 2017:17:e101-e106.

7. Lindsay SW, Wilson A, Golding N, Scott TW, Takken W. Improving the built environment in urban areas to control Aedes aegypti-borne diseases. Bull World Health Organ. 2017;95:607-608.

8. Bradshaw CJ, Leroy B, Bellard C, Roiz D, Albert C, Fournier A, et al. Massive yet grossly underestimated global costs of invasive insects. Nature Communications. 2016;7:12986.

9. Shepard DS, Undurraga EA, Halasa YA, Stan away JD. The global economic burden of dengue: a systematic analysis. Lancet. Infect Dis. 2016;16:935-941.

10. Kuri-Morales P, Correa-Morales F, González-Acosta C, Sánchez-Tejeda G, Dávalos-Becerril E, Juárez-Franco MF, et al. First report of Stegomyia aegypti (Aedes aegypti) in Mexico City, Mexico. Med Vet Entomol. 2017;31:240-242.

11. Yáñez-Arenas $C$, Rioja-Nieto $R$, Martín GA, Dzul-Manzanilla $F$, Chiappa-Carrara X, Buenfil-Ávila A, et al. Characterizing environmental suitability of Aedes albopictus (Diptera: Culicidae) in Mexico based on regional and global niche models. J Med Entomol. 2018;55:69-77.

12. Mejía-Guevara MD, Correa-Morales F, González-Acosta C, Dávalos-Becerril E, Peralta-Rodríguez JL, Martínez-Gaona A, et al. El mosquito del dengue Aedes aegypti en la Ciudad de México. Invasión incipiente y sus potenciales riesgos. Gac Med Mex. 2020;156:388-395.

13. Dávalos-Becerril E, Correa-Morales F, González-Acosta C, Santos-Luna R, Peralta-Rodríguez J, Pérez-Rentería C, et al. Urban and semi-urban mosquitoes of Mexico City: a risk for endemic mosquito-borne disease transmission. PLoS One. 2019;14:e0212987.

14. Singer M, Clair S. Syndemics and public health: reconceptualizing disease in bio-social context. Med Anthropol Q. 2003;17:423-441.

15. Singer, M. Introduction to syndemics: a critical systems approach to public and community health. EE. UU.: Jossey-Bass; 2019.

16. Sánchez-de la Cruz JP, Tovilla-Zárate CA, González-Morales DL, González-Castro TB. Riesgo de sindemia entre COVID-19 y fiebre del dengue en el sur de México. Gac Med Mex. 2020;156:469-473.

17. Nacher M, Dorine M, Gailes M, Flamed C, Rosset D, Rousseau C, et al. Simultaneous dengue and COVID-19 epidemics: Difficult days ahead? PLoS Negl Trop Dis. 2020 Aug 14;14(8):e0008426. DOI: 10.1371/journal. pntd.0008426. eCollection 2020)

18. Secretaría de Salud [Internet]. México: Panorama Epidemiológico de Dengue. Semana epidemiológica 38, 2020. Disponible en: https://www. gob.mx/cms/uploads/attachment/file/579762/Pano dengue 38 2020.pdf

19. Secretaría de Salud de Jalisco [Internet]. Identifica SSJ primeros casos de COVID-19 y dengue en Jalisco. Disponible en: https://ssj.jalisco.gob. $\mathrm{mx} /$ prensa/noticia/9229 